\title{
Electromagnetic Radiation of Simulated Mid-Gap Streamers
}

\author{
J. Montanyà, F. Fabró, \\ Electrical Engineering Department \\ Polytechnic University of Catalonia. Barcelona Tech (UPC) \\ Terrassa (Barcelona) Spain \\ montanya@ee.upc.edu
}

\begin{abstract}
Electromagnetic field from simulated double-headed mid-gap streamers is computed. The effect of the applied electric field is studied by considering electric fields higher than the conventional breakdown threshold ('overvoltage'). The obtained results show a $20 \mathrm{~dB}$ decay at $\sim 3.8 \mathrm{GHz}$ for conventional breakdown electric field, $\sim 35 \mathrm{GHz}$ for ambient electric fields five times the conventional breakdown and $\sim 170 \mathrm{GHz}$ for fields ten times the conventional breakdown electric field. The limitation in nature of the intense electric fields suggest further experiments using a radio receiver at frequencies of more than $10 \mathrm{GHz}$ in order to investigate the origin of the detected $x$-rays in laboratory sparks.
\end{abstract}

Keywords-streamers; radiation; $x$-rays; lighnining; leaders; TGF.

\section{INTRODUCTION}

The nature of lightning is the nature of leaders. A leader is a conductive plasma channel that can develop in low electric field ambient thanks to the intense ionization at its tip. An important feature of a lightning leader is its streamer zone that helps to propagate the leader by providing charge and current. Streamers are less conductive plasmas than leaders presenting filamentary structures. Streamers are short lived. Lightning leaders are very asymmetric. Negative leaders propagate in a step manner allowing to produce very intense electric fields for a short periods of time. For that reason, it is interesting to investigate the behavior of streamers submitted to high electric fields in what we will name here as 'overvoltage'.

On the other hand, not long ago was discover that lightning can be the source of the Terrestrial Gamma ray Flashes [1] and especially its leader processes [2]. At ground, $\mathrm{x}$-ray emissions are associated at the leader step phase [3-5]. These two discoveries promoted the mechanisms of X-ray radiation in laboratory sparks [6-9]. The investigations show that $\mathrm{X}$-ray primary occur before the breakdown in fast voltage impulses. By means short exposure intensified cameras [8] found that x-ray emissions were associated with streamer phase of the discharge instead with the presence of leaders. Recently, it has been found that RF power radiation at microwave range peaks at the time of the detection of $\mathrm{x}$-rays [9]. That coincidence with the optical observations of streamers [8] encouraged us to investigate the electromagnetic spectrum radiation by streamers.

In this paper we theoretically investigate the spectrum radiation of streamers. In order to do that, a numerical model of a mid-gap streamer is computed. The model obtains the electron and ion distribution in time for several applied electric fields. After the electron distributions are obtained at any time, currents and electromagnetic radiation are calculated.

\section{MODEL}

The dynamics of the modelled streamer are described by the following continuity equations representing a fluid description of streamer plasma (e.g. [10]) coupled with the Poisson's equation:

$$
\begin{aligned}
\frac{\partial n_{e}}{\partial t} & =-\nabla \cdot \overrightarrow{v_{e}}+D_{e} \nabla^{2} n_{e}+\left(v_{i}-v_{a}\right) n_{e}-\beta_{e p} n_{e} n_{p}+S_{p h} \\
\frac{\partial n_{p}}{\partial t} & =v_{i} n_{e}-\beta_{e p} n_{e} n_{p}-\beta_{n p} n_{n} n_{p}+S_{p h}(2) \\
\frac{\partial n_{n}}{\partial t} & =v_{a} n_{e}-\beta_{n p} n_{n} n_{p}(3) \\
\nabla^{2} \phi & =-\frac{e}{\varepsilon_{o}}\left(n_{p}-n_{e}-n_{n}\right)
\end{aligned}
$$

where $n_{e}, n_{p}$ and $n_{n}$ are the electron, positive ion and negative ion densities, respectively. The electron drift velocity is $\overrightarrow{v_{e}}=-\mu_{e} \vec{E}$, where $\mu_{e}$ is the absolute value of the electron mobility [11]. $D_{e}$ and $v_{i}$ are the electron diffusion [12] the ionization [13] coefficient, respectively. The attachment coefficient $v_{a}$ is the addition of the two-body and three-body individual coefficients [11]. Recombination coefficients are $\beta_{e p}$ and $\beta_{n p}$ that represents the electron-positive ion recombination and negative-positive ion recombination, respectively [11]. The rate of electron-ion pair production due to photoionization is $S_{p h}$ [12]. In Poisson's equation for the electric potential $\phi, e$ is the absolute value of the charge of the electron and $\varepsilon_{o}$ the permittivity of free space. 
The electric field is calculated by means of the electric potential gradient $\vec{E}=-\nabla \phi$. The Possison's equation is solved by means of the SSOR method [14].

The model of the streamer is simulated for a domain of $2 \mathrm{~cm}$ in the $\mathrm{Z}^{\prime}$-direction with three case of studies of the applied electric field $E_{o}=E_{k}, E_{o}=5 E_{k}$ and $E_{o}=10 E_{k}$ where here $E_{\mathrm{k}}$ is considered $2.7 \times 10^{6} \mathrm{~V} / \mathrm{m}$. It is important to mention that the $5 E_{k}$ and $10 E_{k}$ might not be realistic in nature as it will be discussed in section 4 . The computation of the model is initiated with a Gaussian cloud of plasma of $10^{20} \mathrm{~m}^{-3}$ at the middle of the domain. The model here is $1.5 \mathrm{D}$ [13] considering an effective streamer radius for photoionization of $0.5 \times 10^{-3} \mathrm{~m}$ calculation. The results of the 1.5D model was compared with [15] and [16] obtaining similar results.

The model provides the electron and ion density at any part of the discretized domain at time intervals of $10 \mathrm{ps}$. But in order to calculate the current (moving charges) the drift and diffusion of electrons needs to be computed. The electron flux at each infinitesimal domain interval $\left(d \tau^{\prime}\right)$ at $z^{\prime}$ is calculated by

$$
j_{Z^{\prime}}=-D_{e} \nabla n_{e}-\mu_{e} E n_{e}(5)
$$

Once the charge and currents are determined at any $z$ ' of the domain, the electromagnetic fields can be calculated. Here we calculate the radiated component of electric field by means of the retarded scalar and vector potentials.

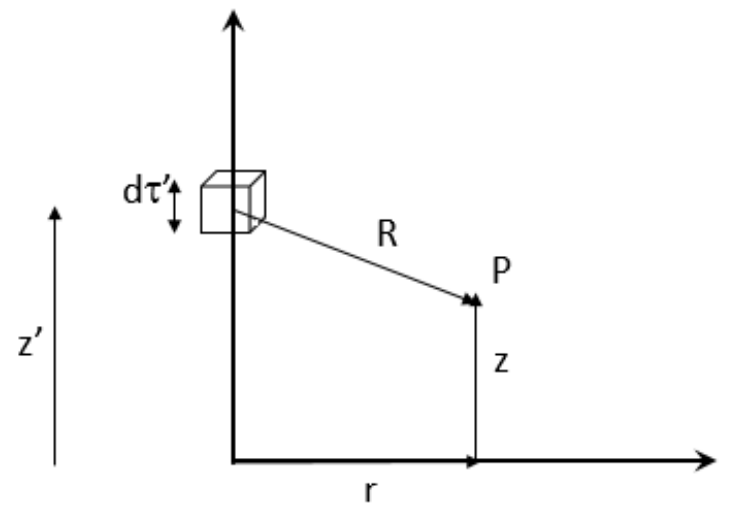

Figure 1. Geometry for calculation the electromagnetic field at point $\mathrm{P}$ produced by a streamer volume current $\mathrm{d} \tau$ '.

For a z'-directed current density element $j_{z}$, in a volume $\tau$ ' at $z^{\prime}$ (Fig. 1), the radiated vertical component of the electric field can be calculated by means of the Jefimenko's equations (e.g. [17]) and the equation (5) resulting in:

$$
d E_{z}(\mathrm{z}, t)=\frac{e d \tau^{\prime}}{4 \pi \varepsilon_{o}}\left[\frac{r^{2}}{R^{3} c^{2}} \frac{\partial^{2}}{\partial t^{2}} j_{z}\left(z^{\prime}, t-\frac{R}{c}\right)\right](6)
$$

where $c$ is the speed of light. We selected only the radiation field. The radiation field is predominant at distances where $\mathrm{r}>>\lambda$.
Equation (6) is numerically integrated considering a streamer radius of $r_{s}=12.5 \mathrm{~mm}$ [15]. The spectrum of the resulted time-domain electric field calculated by means of the fast Fourier transformation (e.g. [18]).

The model described in (1-4) does not include runaway electrons so the radiation produced by the flux of runaway electrons is not computed.

\section{RESULTS}

Our interest result of the streamer model described by equations (1)-(4) is the electron density at any part of the domain. Fig. 2 shows the electron density at different times for the case where $E_{o}=E_{k}$.

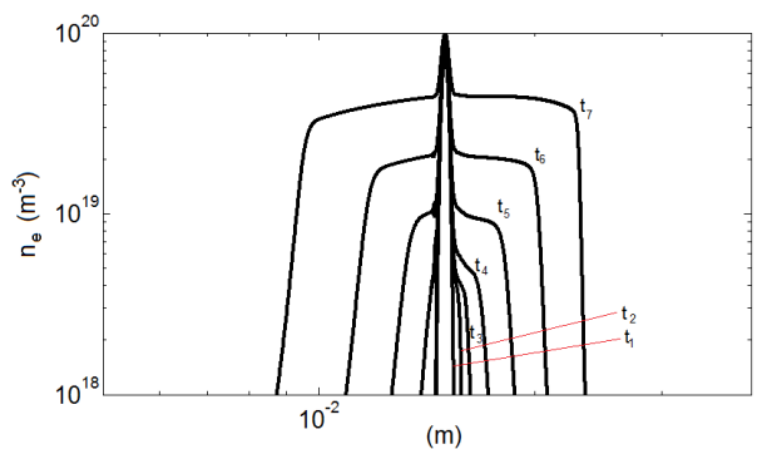

Figure 2. Electron density at different times $\left(t_{1}=0 \mathrm{~ns}, \mathrm{t}_{2}=1 \mathrm{~ns}, \mathrm{t}_{3}=2 \mathrm{~ns}, \mathrm{t}_{4}=3 \mathrm{~ns}\right.$, $\left.\mathrm{t}_{5}=4 \mathrm{~ns}, \mathrm{t}_{6}=5 \mathrm{~ns}, \mathrm{t}_{7}=6 \mathrm{~ns}\right)$ of the simulated streamer with $E_{o}=E_{k}$.

The simulated mid-gap streamer shows bidirectional development (e.g. Fig 2). The propagation to the right corresponds to the negative front whereas the propagation to the left corresponds to the positive front. It can be seen from Fig. 2 how the streamer accelerates as it propagates. The electric field at the streamer fronts also grow as the streamer development (approaching to the simulated electrodes). As pointed by many authors photoionization is one of the main responsible of the speed and acceleration of the streamer.

In order to calculate the radiation field and then its spectrum, the current needs to be calculated. The contribution to the current is due to drift and diffusion of electrons computed by the electron flux equation (5). The flux of electrons at different times of the computed streamer for $E_{o}=E_{k}$ is depicted in Fig. 3 . 


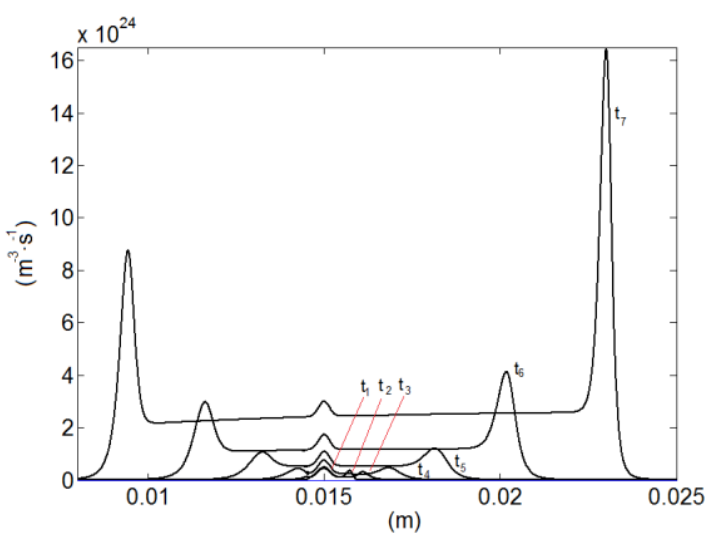

Figure 3. Flux of electrons $j_{z^{\prime}}$ at at different times $\left(\mathrm{t}_{1}=0 \mathrm{~ns}, \mathrm{t}_{2}=1 \mathrm{~ns}, \mathrm{t}_{3}=2 \mathrm{~ns}\right.$, $\left.\mathrm{t}_{4}=3 \mathrm{~ns}, \mathrm{t}_{5}=4 \mathrm{~ns}, \mathrm{t}_{6}=5 \mathrm{~ns}, \mathrm{t}_{7}=6 \mathrm{~ns}\right)$ of the simulated streamer with $E_{o}=E_{k}$.

After the electron flux is computed, the radiated electric field due to the streamer current is calculated by means of equation (6). The spectrum is calculated with the obtained time domain electric field $E_{z}(\mathrm{z}, t)$. The resulted spectrums are presented in Fig. 4.

a)

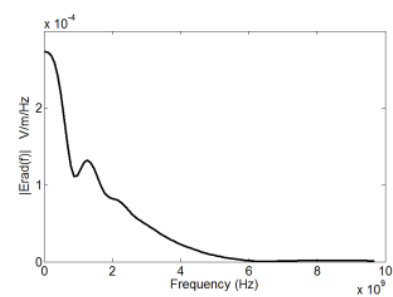

b)

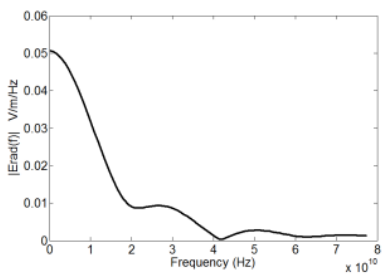

c)

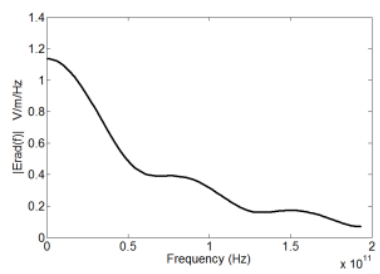

Figure 4. Calculated spectrum of $E_{z}$ for the range of $\mathrm{z}^{\prime}=1.84 \mathrm{~cm}$ to $2.08 \mathrm{~cm}$. a) $\left.\mathrm{E}_{\mathrm{o}}=\mathrm{E}_{\mathrm{k}}, \mathrm{b}\right) \mathrm{E}_{\mathrm{o}}=5 \cdot E_{k}$ and c) $\mathrm{E}_{\mathrm{o}}=10 \cdot \mathrm{E}_{\mathrm{k}}$. Note that the three figures have different axes. The oscillation in the calculated spectrums are due to the truncation of the current.

The radiation electromagnetic spectrums due to the streamer currents in Fig 4 show how the spectrum increases as the applied electric field. For $E_{o}=E_{k}$ a decrease of $20 \mathrm{~dB}$ is found about $\sim 3.8$ $\mathrm{GHz}$, for the case of $E_{o}=5 \cdot E_{k}$ that would reach $\sim 35 \mathrm{GHz}$ and for the case $E_{o}=10 \cdot E_{k}$ that would suppose a $20 \mathrm{~dB}$ decrease at $\sim 170$ $\mathrm{GHz}$. Of course the cases corresponding to $5 \cdot E_{k}$ and $10 \cdot E_{k}$ correspond to a very intense 'over voltage' situation similar to those conditions of nanosecond discharges where x-rays are found [19]. The spectrums presented here show some oscillation due to the current truncation otherwise the computation time would be much larger. The real spectrum would be dominated by the spectrum similar of a Gaussian pulse representing the front of the streamer.

In order to show the influence of the applied electric field ('overvoltage') in the development of the streamer, the speeds of the negative front are calculated and presented in Fig. 5.

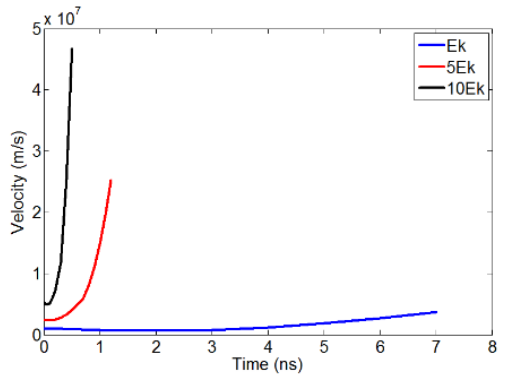

Figure 5. Negative front velocity for the three simulated cases.

The plot in Fig.5 shows how the streamer developing at $E_{o}=E_{k}$ its maximum speed is below $5 \cdot 10^{6} \mathrm{~m} / \mathrm{s}$, consistent with [15]. The speed and acceleration drastically increases as the applied electric field is increased 5 times and one order of magnitude. This change of speed is reflected to the electromagnetic radiation spectrum.

\section{DISCUSSION AND CONCLUSIONS}

In this paper we presented simulation results of the electromagnetic spectrum due to the streamer current for different applied electric fields. The fields over $E_{k}$ would represent an 'overvoltage' case [19] that it might be also produced at the front of negative stepped leader (corona flash [20]). However in reality intense electric fields $\left(>E_{k}\right)$ cannot be persist for a long time because the exponential multiplication of electrons (e.g [21-22]). This time is related to the time of screening of the electric field by charges in a conducting medium (Maxwell relaxation time). Maxwell relaxation timescale is $\varepsilon_{o} / \sigma$, where $\sigma$ is the conductivity of air. Because the conductivity of air exponentially increases for intense electric fields the time in which the electric field can persist decrease inversely. As an example, [22] calculates that at ground pressure, fields of $10 \mathrm{MV} / \mathrm{m}\left(\sim 3 \cdot E_{k}\right)$ would have a timescale not higher than the nanosecond. Fields of about $5 \mathrm{MV} / \mathrm{m}\left(\sim 1.5 \cdot E_{k}\right)$ can be sustained for $\sim 30 \mathrm{~ns}$. In [23] the authors showed that the maximum electric fields at streamer heads in ambient field of $\sim 1.5 \cdot E_{k}$ are $\sim 5 \cdot E_{k}$ occurring just prior branching of the streamer. From these intense electric fields, it is shown that most of the runaway electrons have energies of $\sim 65 \mathrm{keV}$. In case of lighnting, the fields $(\geqslant 600 \mathrm{kV} / \mathrm{m})$ during negative corona flashes would allow further acceleration of these energetic electrons [23] reaching energies in the range of several MeV.

As it has been shown in [9], $\mathrm{x}$-ray detections in laboratory sparks are produced simultaneously with the peak of radiated RF power at microwaves $(2.4 \mathrm{GHz})$. The simulations show how at 
the range of $2.4 \mathrm{GHz}$ the radiation at $E_{o}=E_{k}$ is highly attenuated but still existing. Combining the results of [8], [9] it is shown that the x-rays might be related to the encounter of space streamers. In such encounter, the electrons would highly accelerate similar to 'overvoltage' cases simulated here. That sounds reasonable to attribute the peak found in electromagnetic radiation in [9] to be maximum at the time of the encounter. The question is if the electromagnetic radiation and x-rays share the same mechanism due to intense electric field at the streamer tip with the limited 'overvoltage'. The limit in the ambient electric field ('overvoltage') suggests reproducing the experiment in [9] with the receiver tuned higher frequencies in order to investigate if the radiation mechanism is due to the streamer current or by Bremsstrahlung radiation.

\section{ACKNOWLEDGMENT}

This work was supported by research grants from the Spanish Ministry of Economy and Competitiveness (MINECO) and the European Regional Development Fund (FEDER): (MINECO) AYA2011-29936-C05-04; (MINECO/FEDER) ESP2013- 48032-C5-3-R and (MINECO/FEDER) ESP201569909-C5-5-R. We thank M. Arrayás and J.-L. Trueba for the discussions related to this work.

\section{REFERENCES}

[1] G. J., Fishman, Bhat P.N., Mallozzi R., Horack J.M., KoshutT., Kouveliotou C., Pendleton G.N., Meegan C.A., Wilson R.B., Paciesas W.S., Goodman S.J., Christian H.J., "Discovery of intense gamma-ray flashes of atmospheric origin," Science 264, pp. 1313-1316, 1994.

[2] E. Williams, et al., "Lightning flashes conducive to the production and escape of gamma radiation to space," J. Geophys. Res., 111, D16209, doi:10.1029/2005JD006447, 2006.

[3] C.B. Moore, K. B. Eack, G. D. Aulich, and W. Rison, "Energetic radiation associated with lightning stepped-leaders," Geophys. Res. Lett., 28, pp. 2141-2144, 2001.

[4] J. R. Dwyer, et al., "X-ray bursts associated with leader steps in cloud-toground lightning," Geophys. Res. Lett., 32, L01803, doi:10.1029/2004GL021782, 2005.

[5] J. Montanyà, F. Fabró, O. van der Velde, D. Romero, G. Solà, J. R. Hermoso, S. Soula, E. R. Williams, and N. Pineda, "Registration of Xrays at $2500 \mathrm{~m}$ altitude in association with lightning flashes and thunderstorms," J. Geophys. Res. Atmos., 119, pp. 1492-1503, 2014.

[6] J. R. Dwyer, H. K. Rassoul, Z. Saleh, M. A. Uman, J. Jerauld, and J. A. Plumer (2005b), "X-ray bursts produced by laboratory sparks in air," Geophys. Res. Lett., 32, L20809, doi:10.1029/2005GL024027, 2005

[7] V. March and J. Montanyà," Influence of the voltage-time derivative in X-ray emission from laboratory sparks," Geophys. Res. Lett., 37,L19801, doi:10.1029/2010GL044543, 2010.

[8] P. Kochkin, A.P.J. van Deursen, and U. Ebert, "Experimental study on hard x-rays emitted from metre-scale negative discharges in air," Journal of Physics D: Applied Physics, 48(2), 025205-1/13, 2015.

[9] J. Montanyà J., F. Fabró, V. March, O. van der Velde, G. Sola, D. Romero and $\mathrm{O}$. Argemí, X-rays and microwave RF power from high voltage laboratory sparks, Journal of Atmospheric and Solar-Terrestrial Physics, doi:10.1016/j.jastp.2015.06.009, 2015.
[10] N.Y. Babaeva, and G. V. Naidis,"Dynamics of positive and negative streamers in air in weak uniform electric fields," IEEE Trans. Plasma Sci., 25, 375, doi:10.1109/27.602514, 1997.

[11] X.M. Zhao, J. C. Diels, C. Y. Wang, and J. M. Elizondo ," Femtosecond ultraviolet laser pulse induced lightning discharge in gases," IEEE J. Quantum Electr., 31, 599-612, 1995.

[12] A.A. Kulikovsky, "The role of photoionization in positive streamer dynamics," J. Phys. D Appl. Phys., 33, 1514-1524, 2000.

[13] E.M. Bazelyan, Yu. P. Raizer. Spark Discharge. CRC Press, Boca Raton, New York, 1998

[14] R. W. Hockney and J. W. Eastwood, Computer Simulation Using Particles, McGraw-Hill, New York, 1988.

[15] N. Liu, and V. P. Pasko (2004), Effects of photoionization on propagation and branching of positive and negative streamers in sprites, J. Geophys. Res., 109, A04301, doi:10.1029/2003JA010064.

[16] A. Bourdon, V. P. Pasko, N. Y. Liu, S. Célestin, P. Ségur and E. Marode, "Efficient models for photoionization produced by non-thermal gas discharges in air based on radiative transfer and the Helmholtz equations," Plasma Sources Sci. Technol. 16, pp. 656-678, 2007.

[17] D. J. Griffiths, "Introduction to Electrodynamics," Prentice Hall, 3rd edition, 1999 .

[18] A. V. Oppenheim and R. W. Schafer, Discrete-time Signal Processing, Prentice-Hall signal processing series, 1988.

[19] L. P. Babich and T. V. Loiko, "Subnanosecond Pulses of Runaway Electrons Generated in Atmosphere by High_Voltage Pulses of Microsecond Duration," Doklady Physics, Vol. 54, No. 11, pp. 479-482, 2009.

[20] E. M. Bazelyan, and Y. P. Raizer, Lightning Physics and Lightning Protection, IoP Ltd., Bristol, U. K., 2000.

[21] M.I. Dyakonov and V. Y. Kachorovskii, "Theory of streamer discharge in semiconductors," Sov. Phys. JETP, Engl. Transl., 67, 1049, 1988.

[22] S. Celestin, and V. P. Pasko, "Energy and fluxes of thermal runaway electrons produced by exponential growth of streamers during the stepping of lightning leaders and in transient luminous events,. J. Geophys.Res., 116, A03315, doi:10.1029/2010JA016260, 2011. 